\title{
Desmoid-type fibromatosis of the chest wall: a case report
}

\author{
Yi Xiao, Jinyuan He, Chulian Gong, Libao Liu, Shaohong Huang \\ The Third Affiliated Hospital of Sun Yat-sen University, Guangzhou, China \\ Correspondence to: Shaohong Huang; Libao Liu. No. 108, Tianhe Road, Tianhe District, Guangzhou, China. \\ Email: legendhuang@126.com; 1152407333@qq.com.
}

\begin{abstract}
Desmoid-type fibromatosis (DF), also known as deep fibromatosis or desmoid tumor, is an extremely rare neoplasm that develops from fascia and musculoaponeurotic tissue. These tumors are characterized by slow progressive growth, local invasion, and local recurrence after surgical excision, but they lack metastatic potential. DF accounts for $3.5 \%$ of all fibrous tumors, with an annual incidence of approximately $2-4 /$ million. Until now, only a small number of cases have been found in the chest wall. Herein, we present a rare case of chest wall DF in a 43 -year-old female, which was discovered accidentally due to a thoracic wall mass that extended outward from the sternum. Computed tomography scans revealed a subcutaneous soft tissue mass anterior to the sternum, which was considered to be a mesenchymal tumor or an inflammatory lesion. The patient underwent surgical excision of the mass. The mass was completely removed and all margins were negative. According to the pathological results, the patient was finally diagnosed as DF. Postoperative radiotherapy was suggested subsequently, especially considering the locally aggressive and infiltrative nature of the tumor. However, this was rejected by the patient, and biannual reexamination was recommended instead. Despite the absence of postoperative radiotherapy, there was no evidence of local recurrence 2 years later. We consider regular postoperative follow-up may be able to replace postoperative radiotherapy, and if there exist an opportunity to completely resect the mass, surgical is a worthwhile choice.
\end{abstract}

Keywords: Desmoid-type; fibromatosis; thoracic wall

Submitted Jun 05, 2020. Accepted for publication Sep 11, 2020.

doi: $10.21037 / \mathrm{atm}-20-5237$

View this article at: http://dx.doi.org/10.21037/atm-20-5237

\section{Introduction}

Desmoid-type fibromatosis (DF), also known as deep fibromatosis or desmoid tumor, is an extremely rare neoplasm that develops from fascia and musculoaponeurotic tissue. DF accounts for approximately $3.5 \%$ of all fibrous tumors, with an annual incidence of approximately $2-4 /$ million $(1,2)$. It can occur in almost any anatomical region but is most typically found in the abdomen, and it is especially common in females of childbearing age (3). The tumors are characterized by slow progressive growth, local invasion, and local recurrence after surgical excision; however, they do not exhibit metastatic potential. The pathogeny of DF is diverse and uncertain, main causes including traumatic, abnormal level of estrogen and genetic factors, surgery is considered the most common cause (2).
Previous studies have shown that patients with familial adenomatous polyposis (FAP) are at a significantly higher risk (approximately 800 -fold) of developing DF (4). DF is discovered almost everywhere in the body. DF present as, painless, hard-textured, infiltrative masses, which is often described as ill-defined masses with a fibrous component on CT examination. Pathological diagnosis is the "golden standard" diagnosis of DF. Microscopically, abundant spindle cells with typical small nuclei are filled with collagen. The immunophenotype display MSA and SMA are positive, desmin, h-caldesmon, and S-100 are negative (5). DF with FAP, which accounts for $5-10 \%$ of all DF cases, is most prevalent intra-abdominally (4). In comparison, sporadic lesions are more likely to occur in the hip/buttock area, extremities, and shoulders (6). Studies have reported 


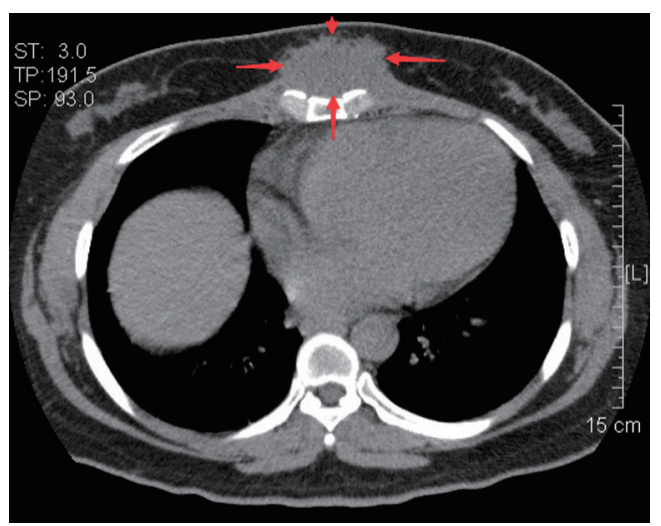

Figure 1 CT scan of the chest (mediastinal window). Subcutaneous soft tissue mass in front of the sternum (level of 7-9 vertebra): the boundary between the mass and sternum is slightly unclear, with no destruction of the sternum.

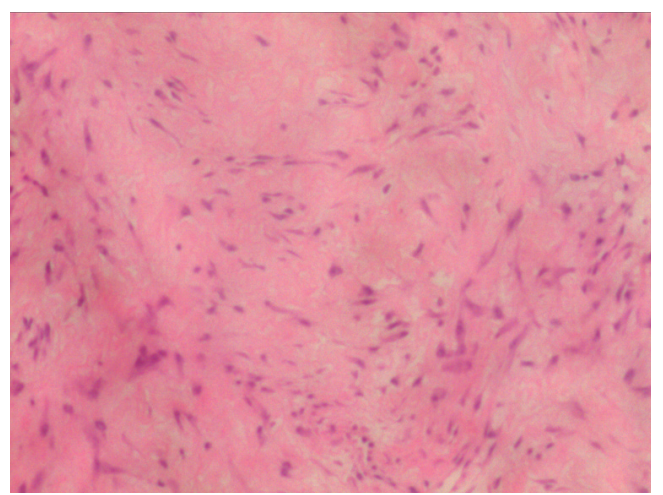

Figure 2 Haematoxylin-eosin staining (frozen section). Hyperplastic spindle cells (mild atypia and scarce mitoses) arranged in a fascicular or interwoven pattern with an unclear boundary from adipose tissue. $\mathrm{HE} \times 200$.

that the $\beta$-catenin pathway plays a critical role in sporadic lesions, with dysfunction of the CTNNB1 gene (2). DF of the chest wall, which is extra-abdominal, accounts for only $10-20 \%$ of all fibromatosis cases (7). Currently, there is varied clinical treatments for DF, including surgical treatment, drug treatment (mainly includes cytotoxic chemotherapy, targeted therapies, NSAIDs, and hormonal therapy), and radiotherapy (5). Even so, owing to the locally aggressive and infiltrating nature of tumor, the possibility of local recurrence is extremely high, and expand the extent of surgical to ensure complete resection in DF which is a kind of disease with low degree of malignancy is probably unacceptable, especially in critical sites (such as the limb girdles, head, and neck). Hence it may be considerable difficult to draw up an appropriate treatment regimen to achieve a satisfactory therapeutic effect. We present the following article in accordance with the CARE reporting checklist (available at http://dx.doi.org/10.21037/atm-205237).

\section{Case presentation}

A 43-year-old woman presented with a 1-month history of a chest wall mass that extended outward from the sternum first visited the Department of Thoracic Surgery of the Third Affiliated Hospital of Sun Yat-sen University. Physical examination found a firm and painless 8-9 cm mass on the sternum, with no swelling of superficial lymph nodes. The patient had no history of surgery, trauma, or FAP, or other significant family history. Computed tomography (CT) scans revealed a subcutaneous soft tissue mass anterior to the sternum, which was considered to be a mesenchymal tumor or an inflammatory lesion (Figure 1). After discussing the case during a multidisciplinary conference, and obtaining the patient's consent, surgery was chosen to resect the mass, along with frozen section examination to analyze its characteristics. Intraoperatively, a dense mass measuring $6 \mathrm{~cm} \times 6 \mathrm{~cm} \times 3 \mathrm{~cm}$ was identified on the sternum; it was lacking a capsule and there was no invasion of the sternum. The mass was completely excised and sent for frozen section examination. Microscopic evaluation revealed possible DF with negative margins (Figure 2). Accordingly, extensive resection of the sternum and adjacent soft ribs was cancelled. Immunostains reported S100 (-), SMA (+), Desmin (partly +), CD34 (+), Bcl-2 (-), B-Catenin (partly +), $\mathrm{Ki}-67(5 \%+)$. The results of pathological examination (Figure 3) were consistent with the frozen section, and the patient was diagnosed with DF. During the postoperative multidisciplinary conference, postoperative radiotherapy was suggested, especially considering the locally aggressive and infiltrative nature of the tumor. However, this was rejected by the patient, and biannual re-examination was recommended instead. Despite the absence of postoperative radiotherapy, there was no evidence of local recurrence 2 years later.

All procedures performed in studies involving human participants were in accordance with the ethical standards of the institutional and/or national research committee(s) and with the Helsinki Declaration (as revised in 2013). 


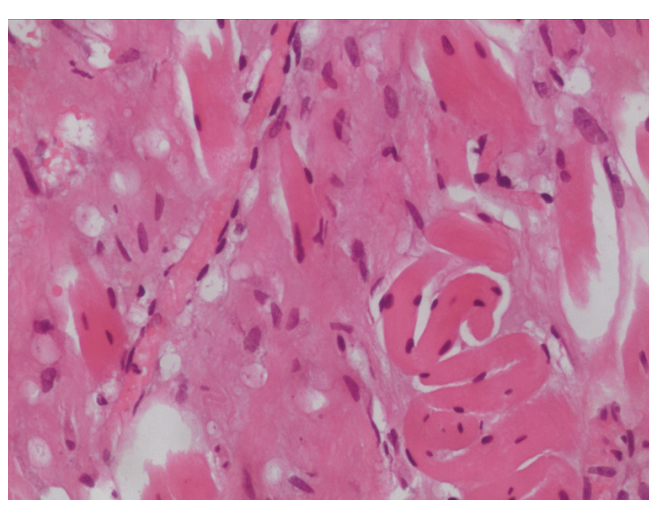

Figure 3 Haematoxylin-eosin staining (pathological examination after operation). The mass consisted of spindle cells (mild atypia and scarce mitoses) arranged in a fascicular or interwoven pattern with an unclear boundary from adipose tissue and striated muscle tissue. $\mathrm{HE} \times 400$.

\section{Discussion}

Considering the variable nature of DF, including the unpredictable phases of its proliferation and stabilization, as well as the rate of regression without any therapy, the European Organization for Research and Treatment of Cancer recommends the "wait and see" approach as an initial strategy (8). Furthermore, according to the previous report, the 5-year progression-free survival rate when employing the "wait and see" approach is not significantly different to that where medical therapies have been utilized (49.9\% and 58.6\%, respectively) (9). Moreover, resection of a tumor with negative margins can be difficult due to its locally aggressive and infiltrative nature, especially in critical sites (such as the limb girdles, head, and neck).

Surgery is considered a potentially curative treatment (10), although it is important that surgical treatment only be considered when complete removal of tumors with negative margins can be achieved without causing critical functional defects. Complete control of DF is difficult to achieve. Despite the lack of metastatic potential and favorable survival prognosis, DF has an approximate local recurrence rate of $20-65 \%$ within 5 years (11). Previous studies regarding DF with negative margins have shown that the local control rate of surgery with radiotherapy is not significantly different from that of surgery alone $(75 \%$ and $72 \%$, respectively) (12). However, radiotherapy (with a recommended dose of 50-56 Gy) is necessary for patients with positive margins or local recurrence (5). Furthermore, cytotoxic chemotherapy is considered as a useful strategy when tumors are rapidly growing or are located in critical sites (5). Some NSAIDs, such as sulindac, have been shown to induce regression of these tumors by regulation of the $\beta$-catenin pathway $(13,14)$.

Factors such as age (<37 years), tumor size $(>7 \mathrm{~cm})$, positive margins, and extra-abdominal localization are presumed to be poor prognostic indicators for DF (15). While necessary, microscopic negative margins are difficult to achieve, especially considering the locally aggressive and infiltrative nature of the tumor. In a classical wide radical resection, excision of the adjacent bony tissue of a tumor is regarded as a common treatment strategy. However, in this case, complete removal of a tumor with microscopic negative margins was realized, and the adjacent sternum and costal cartilage were preserved. Furthermore, despite the absence of postoperative radiotherapy, CT scan reexamination revealed no signs of local recurrence. Thus, for patients with complete resection with negative margins, regular follow-up may be considered a viable alternative to postoperative radiotherapy. Meanwhile, under conditions where complete resection is possible and without loss of vital function, like this case, surgical resection may be preferred, instead of just "wait and see". The unpredictability of DF may result in a loss of opportunity to obtain a negative margin and lead to a broader extent of resection. However, the limitation in this case was the insufficient follow-up time (2 years).

\section{Acknowledgments}

Funding: None.

\section{Footnote}

Reporting Checklist: The authors have completed the CARE reporting checklist. Available at http://dx.doi.org/10.21037/ atm-20-5237

Conflicts of Interest: All authors have completed the ICMJE uniform disclosure form (available at http://dx.doi. org/10.21037/atm-20-5237). The authors have no conflicts of interest to declare.

Ethical Statement: The authors are accountable for all aspects of the work in ensuring that questions related to the accuracy or integrity of any part of the work are appropriately investigated and resolved. All procedures performed in studies involving human participants were in 
accordance with the ethical standards of the institutional and/or national research committee(s) and with the Helsinki Declaration (as revised in 2013). Written informed consent was obtained from the patient for publication of this manuscript and any accompanying images.

Open Access Statement: This is an Open Access article distributed in accordance with the Creative Commons Attribution-NonCommercial-NoDerivs 4.0 International License (CC BY-NC-ND 4.0), which permits the noncommercial replication and distribution of the article with the strict proviso that no changes or edits are made and the original work is properly cited (including links to both the formal publication through the relevant DOI and the license). See: https://creativecommons.org/licenses/by-nc-nd/4.0/.

\section{References}

1. Reitamo JJ, Hayry P, Nykyri E, et al. The desmoid tumor. I. Incidence, sex-, age- and anatomical distribution in the Finnish population. Am J Clin Pathol 1982;77:665-73.

2. Jin Z, Zhang Q, Tang D, et al. Successful treatment of giant mesenteric fibromatosis with surgery and tamoxifen: case report. Transl Cancer Res 2019;8:996-1000.

3. Walker EA, Petscavage JM, Brian PL, et al. Imaging features of superficial and deep fibromatoses in the adult population. Sarcoma 2012;2012:215810.

4. Nieuwenhuis MH, Casparie M, Mathus-Vliegen LM, et al. A nation-wide study comparing sporadic and familial adenomatous polyposis-related desmoid-type fibromatoses. Int J Cancer 2011;129:256-61.

5. Martinez Trufero J, Pajares Bernad I, Torres Ramon I, et al. Desmoid-Type Fibromatosis: Who, When, and How to Treat. Curr Treat Options Oncol 2017;18:29.

6. Schlemmer M. Desmoid tumors and deep fibromatoses.

Cite this article as: Xiao Y, He J, Gong C, Liu L, Huang S. Desmoid-type fibromatosis of the chest wall: a case report. Ann Transl Med 2020;8(20):1322. doi: 10.21037/atm-20-5237
Hematol Oncol Clin North Am 2005;19:565-71, vii-viii.

7. Kabiri EH, Al Aziz S, El Maslout A, et al. Desmoid tumors of the chest wall. Eur J Cardiothorac Surg 2001;19:580-3.

8. Kasper B, Baumgarten C, Bonvalot S, et al. Management of sporadic desmoid-type fibromatosis: a European consensus approach based on patients' and professionals' expertise - a sarcoma patients EuroNet and European Organisation for Research and Treatment of Cancer/Soft Tissue and Bone Sarcoma Group initiative. Eur J Cancer 2015;51:127-36.

9. Bonvalot S, Eldweny H, Haddad V, et al. Extra-abdominal primary fibromatosis: Aggressive management could be avoided in a subgroup of patients. Ejso 2008;34:462-8.

10. Wong SL. Diagnosis and management of desmoid tumors and fibrosarcoma. J Surg Oncol 2008;97:554-8.

11. Bonvalot S, Desai A, Coppola S, et al. The treatment of desmoid tumors: a stepwise clinical approach. Ann Oncol 2012;23 Suppl 10:x158-66.

12. Nuyttens JJ, Rust PF, Thomas CR Jr, et al. Surgery versus radiation therapy for patients with aggressive fibromatosis or desmoid tumors: A comparative review of 22 articles. Cancer 2000;88:1517-23.

13. Tanaka K, Yoshikawa R, Yanagi H, et al. Regression of sporadic intra-abdominal desmoid tumour following administration of non-steroidal anti-inflammatory drug. World J Surg Oncol 2008;6:17.

14. Signoroni S, Frattini M, Negri T, et al. Cyclooxygenase-2 and platelet-derived growth factor receptors as potential targets in treating aggressive fibromatosis. Clin Cancer Res 2007;13:5034-40.

15. Salas S, Dufresne A, Bui B, et al. Prognostic factors influencing progression-free survival determined from a series of sporadic desmoid tumors: a wait-and-see policy according to tumor presentation. J Clin Oncol 2011;29:3553-8. 